\title{
Use of LC-Filters to Protect Equipment from Electromagnetic Pulse: Is It Real Necessity or "Business as Usual"?
}

\author{
Vladimir Gurevich, Ph.D.
}

\author{
Central Electrical Laboratory Israel Electric Corp. Haifa, Israel
}

\begin{abstract}
Use of special LC-filters to protect electric and electronic equipment from a devastating impact of electromagnetic pulse is deemed to be a common means of protection described in various standards, reports and articles. This article questions the soundness of the common approach and suggests varistors and suppressors as the main means of protection, thus making protection of industrial equipment much simpler and cheaper.
\end{abstract}

Keywords: LC-filters, EMC, HEMP, electromagnetic pulse

\section{INTRODUCTION}

Protection of electrotechnical and electronic equipment from High Altitude Electromagnetic Pulse (HEMP) impact and Intentional Destructive Electromagnetic Interferences (IDEI) has now become very relevant due to the expanding application of microelectronics in basic fields of engineering, (e.g. power, water supply, communication, etc.) that constitutes the foundation of the country's infrastructure on the one hand, and success in reproduction of extra-power destructive electromagnetic fields - on the other hand [1].

The basic means of electric equipment protection from HEMP, which are capable of creating an electric field with the density of up to $50 \mathrm{kV} / \mathrm{m}$ at the ground surface, are well known and have been used in military systems for a long time. These means include filters, shielded cables, metal shielding shells (Faraday cages) and pulse voltage limiters. The same devices are also recommended as protection of critical industrial and power equipment [2, 3].

\section{LC-Filters as the Main Protection from HeMP}

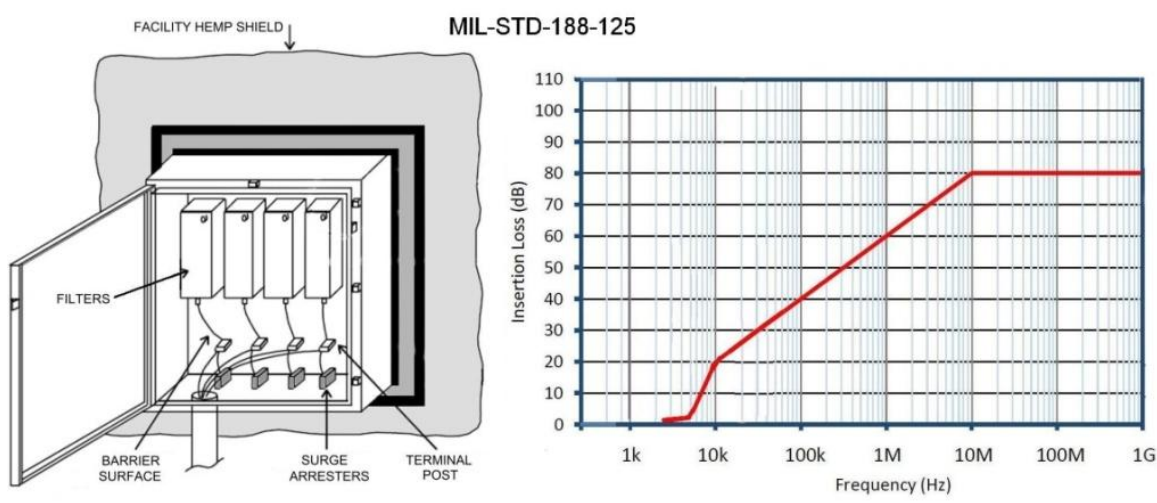

Fig. 1. A standard way of external cables' connection to protected equipment via filters and the required specifications of these filters (MIL-STD-188-125).

The LC-filters prevent penetration from HEMP into the critical equipment among the above mentioned protection means. They are considered to be the main protection means and thus they are separately addressed in military and International Electrotechnical Comission standards For example, MIL-STD-188-125 [4] suggests how to connect external cables to the protected equipment, Fig. 1. Special LC-filters protecting electric and electronic equipment from HEMP and IDEI are now considered so usual and mandatory that nobody questions the soundness of their use. Today, dozens of industrial enterprises produce hundreds of types of these filters, Fig. 2. 

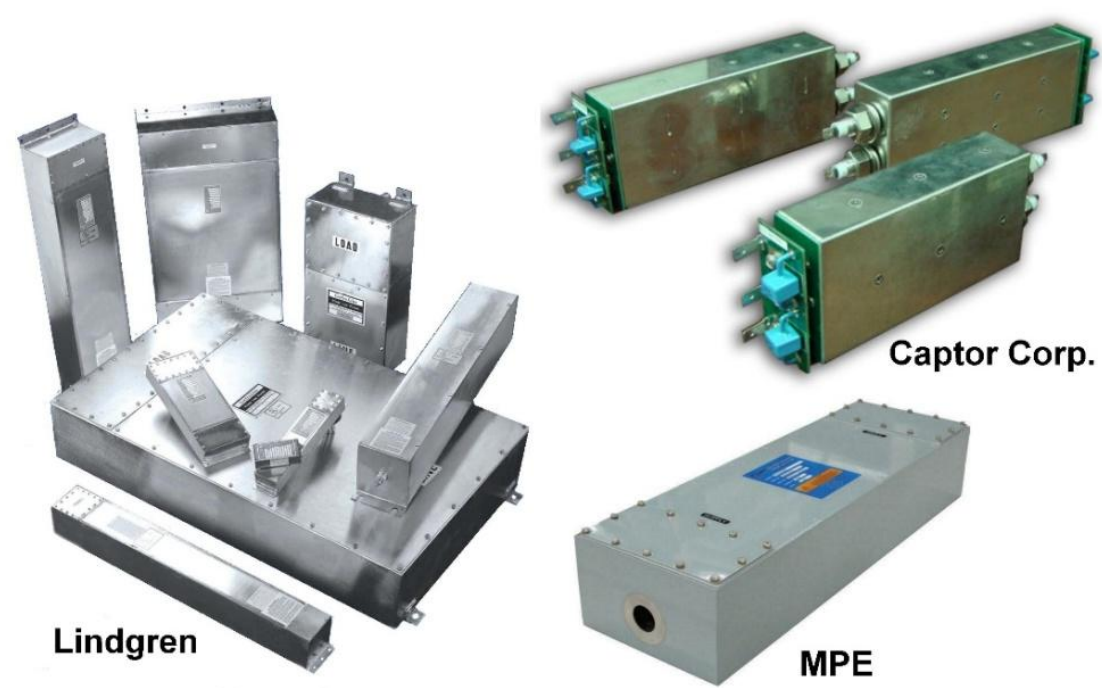

Fig2. Some types of HEMP filters produced by various companies

Moreover, the scope of these filters is not limited to just electronic communication, management and control equipment. They can also be used for high power equipment, Fig. 3.

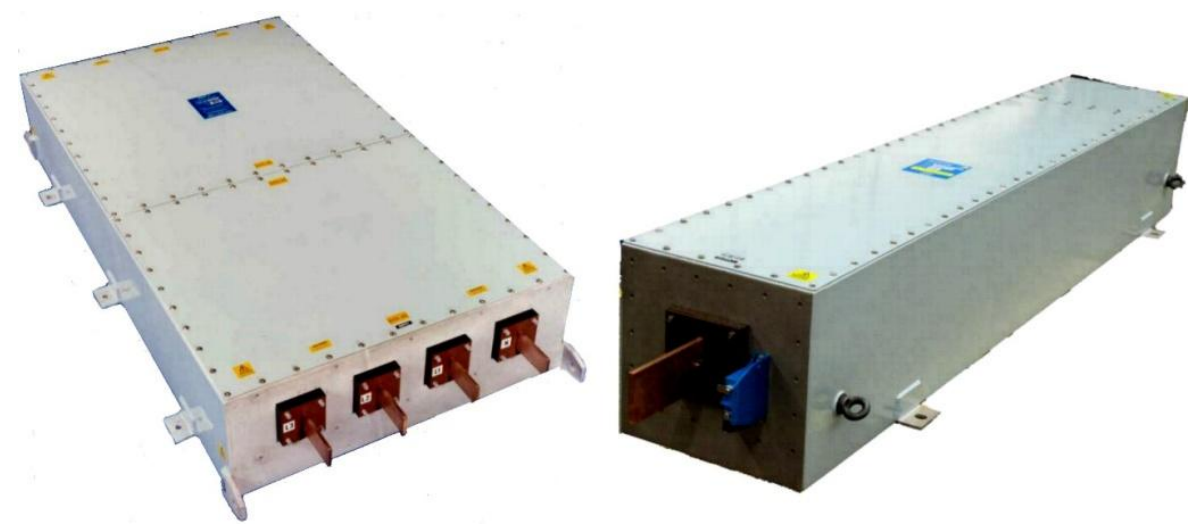

Fig3. Powerful HEMP filters for power circuits rated 800 and 1,200 A

This approach to the filters' use in various types of HEMP-protected equipment and the availability of different types of filters in the market (including those produced in the shape of a wall bushing, filters built-in into multi-pin connectors, etc.) make the developer of protected equipment realize the necessity of these filters. Consequently, diesel generators equipped with high power HEMP filters now exist, Fig. 4.

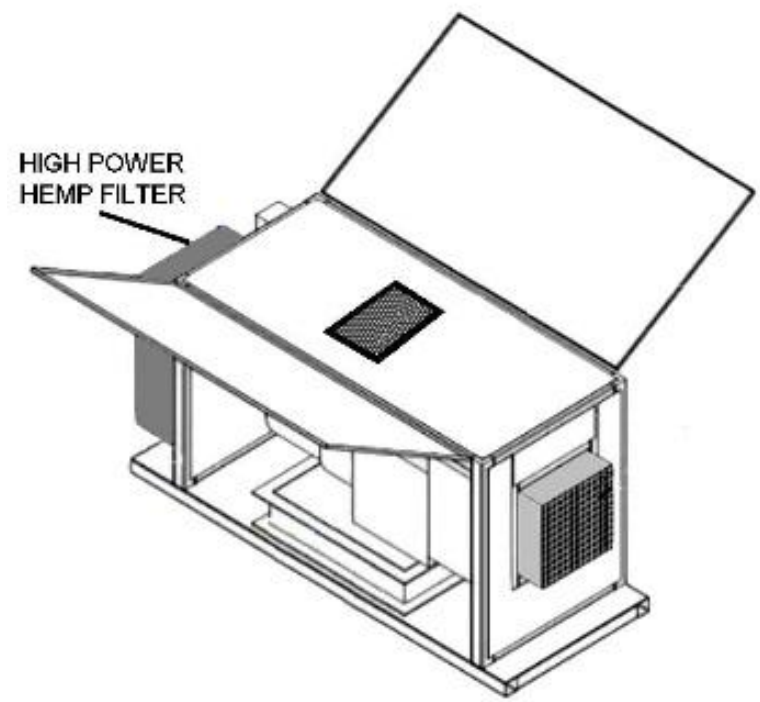

Fig4. Diesel generators produced by EMP Engineering equipped with a powerful HEMP filter. 


\section{Hemp Features that Affect the Choice of Protecting Equipment}

So, how do these filters protect equipment from HEMP? In order to address this issue we need to clearly understand what HEMP is, Fig. 5.
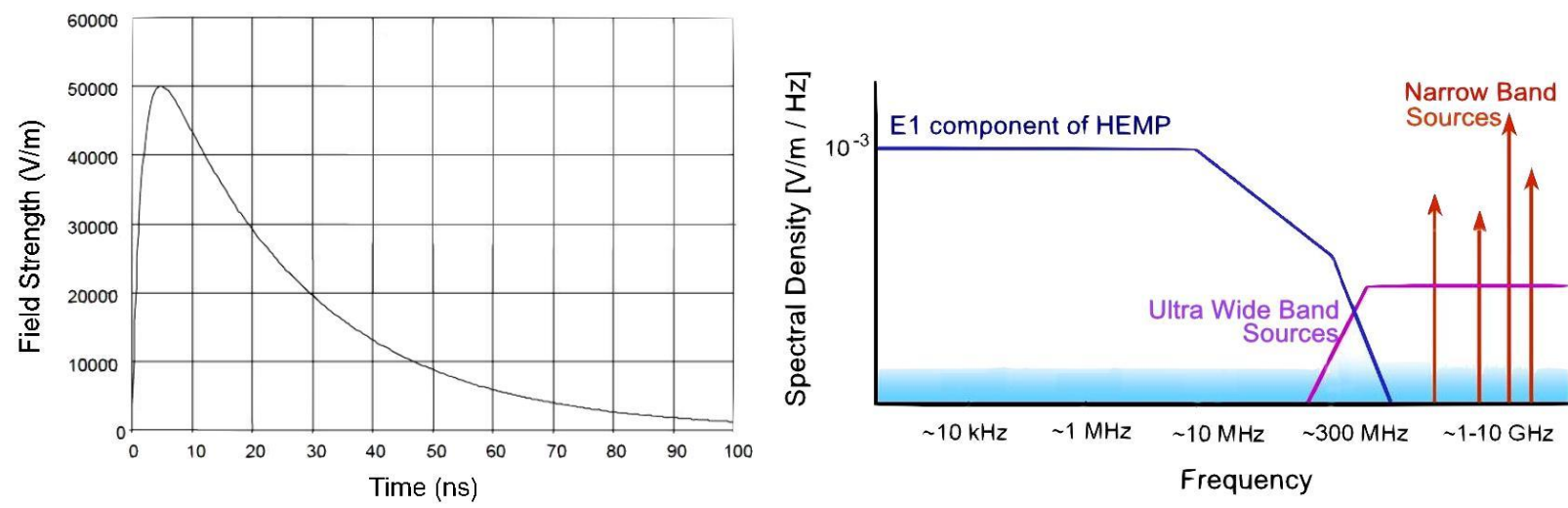

Fig5. Parameters of HEMP pulse (its E1 component) according to IEC 61000-2-9, IEC 61000-2-10, IEC 61000-2-11, IEC 61000-2-13 and MIL-STD-461F.

Having analyzed the specifications shown in Fig. 5, it is obvious that HEMP is a composite interference affecting equipment by high voltage and high frequency. Consequently, HEMP filters need to protect equipment both from HV pulse and high-frequency interference impacts. However, the majority of HEMP filters have limited operating voltage that does not exceed several hundreds of volts, whereas the HEMP's voltage amplitude can reach as high as $50 \mathrm{kV}$. This means that the filters will inevitably be damaged if they are directly connected into the HEMP-affected electric circuit. That is why the filters should be equipped with additional elements that protect from high amplitude pulse overload. The MIL-STD-188-125 stipulates the use of these protecting elements in combination with the filters, Fig. 1. Moreover, some filters are equipped with these protecting elements, mounted inside or outside the case.
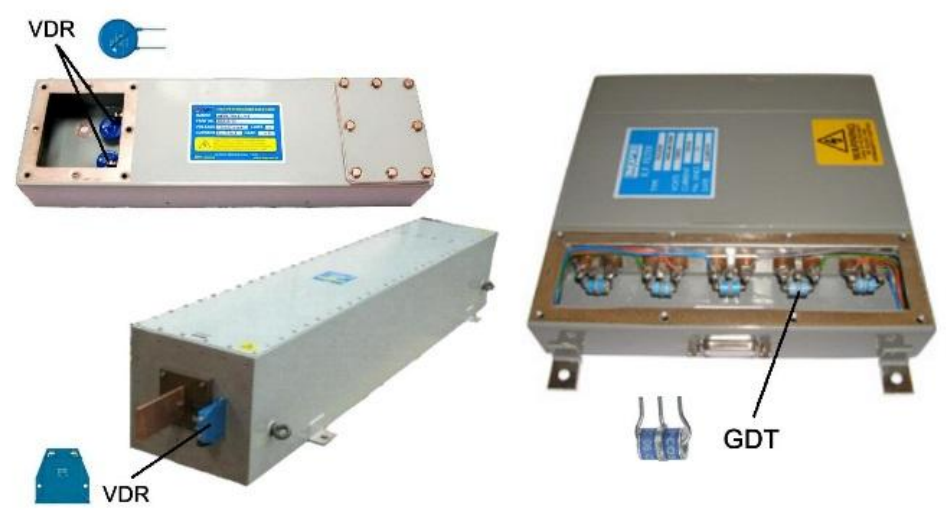

Fig6. Some HEMP filters equipped with HV pulse protecting elements. VDR - varistor, GDT - gas-discharge tube.

We can see similar protecting elements on filters produced by Captor Corp., Fig. 2.

The above mentioned seems to be very consistent and confirms the common approach to HEMP protection based on the widespread use of filters. However, considering that HV pulse and highfrequency interferences represent absolutely different types of impact, (based on their physical properties) and protection from them is ensured by absolutely different technical means, the soundness of the common opinion regarding the necessity of the widespread use of filters as the main HEMP protection is questionable.

These doubts only become stronger after reading researchers and HEMP filter manufacturers' statements :

- Filters are usually rated for hundreds of volts, but an EMP-induced signal may be many thousands of volts" [5] 
- "High Voltage HEMP Filters are designed to protect sensitive electronics equipment during hazardous transient conditions. They are employed to absorb a potentially destructive overshoot voltage" [6]

- "Transient suppressors within HEMP filters are usually varistors" [7].

- "All the lines feature high energy varistor transient suppressors" [8]

- "All lines are individually filtered and featured necessary varistor transient suppressors [9]

Some manufacturers, such as: Huber+Suhner, Meteolab, NexTec, Teledartner and others, equipped their HEMP grade filters with GDT instead of varistors, but according to [10]: "GDT device cannot protect any penetrating EMP noise. In other words, it is not suitable if we use GDT device to protect electronic systems against EMP", therefore such filters are definitely not a solution for HEMP protection.

Let us discuss both aspects (high voltage transient and high frequency impacts) separately.

\section{Protection of Equipment From High-Frequency InTERFEREnCE Generated by HEMP}

As known, all kinds of serious integral circuits (IC) intended for use in military and critical industrial applications must be tested on susceptibility to high frequency interferences according to group of standards IEC 62132, on radiated and conducted disturbances by different methods in the frequency range $150 \mathrm{kHz}$ to $1 \mathrm{GH}$. The controllers and other electronic devices that include IC also must be tested to electromagnetic compatibility according to a group of standards IEC 61000. It is the reason for examination of the damage effect of the microcontroller during the HEMP event [11]. It is very interesting that in this paper the damage effect caused by impact of a high voltage pulse of the HEMP, only without frequency influence, is examined.

It is known that modern industrial and power equipment undergoes compliance checks with the requirements of electromagnetic compatibility (EMC) standards. These standards provide for testing of equipment's resilience to high-frequency, high voltage interferences, applied to equipment inputs (between different inputs and between integrated inputs and the body). For example, IEC 61000-4-12 stipulates equipment checks by applying a high-frequency voltage of $1 \mathrm{MHz}$, and the amplitude of 2.5 $\mathrm{kV}$. IEC 61000-4-4 and IEC 61000-4-5 stipulate application of short pulses (i.e. high-frequency signal) with the amplitude of up to $4 \mathrm{kV}$ to the circuits of the equipment being checked. IEC 61000-43 stipulates the checks of equipment resilience to high-frequency interferences in the frequency range of up to $2 \mathrm{GHz}$. In other words, as for high-frequency interference, the standard requirements shall ensure equipment resistance to these types of interference, including HEMP-generated interference. Some parameters of HEMP-generated pulse interference differ from those that are modeled during equipment EMC compliance tests. Indeed, the HEMP interference pulse is much shorter than the standard pulse used in common EMC tests. However, it is very unlikely that the relay protection, control and automation equipment, resistant to interference under the EMC standard, will fail under the HEMP-generated very short pulse. Moreover, the difference between such impact parameters for HEMP as EFT (Electrical Fast Transient) "quick pulse" resistance testing and EFT parameters of ordinary EMC tests is just the amplitude only of test pulses.

Thus, the appropriateness of special LC-filters protecting equipment from HEMP-generated highfrequency interference is very questionable.

\section{Protection of Equipment from Hemp-Generated High Voltage Puilse}

The situation with HEMP-generated high amplitude voltage pulse applied to equipment circuits is different. The EMC requirements and the applied methods of compliance check are not even close to real overload levels that will affect the equipment under HEMP impact. However, there are wellknown and widely used methods and technical means that ensure protection from high voltage pulses. These include zinc-oxide varistors, for example, Fig. 7. 


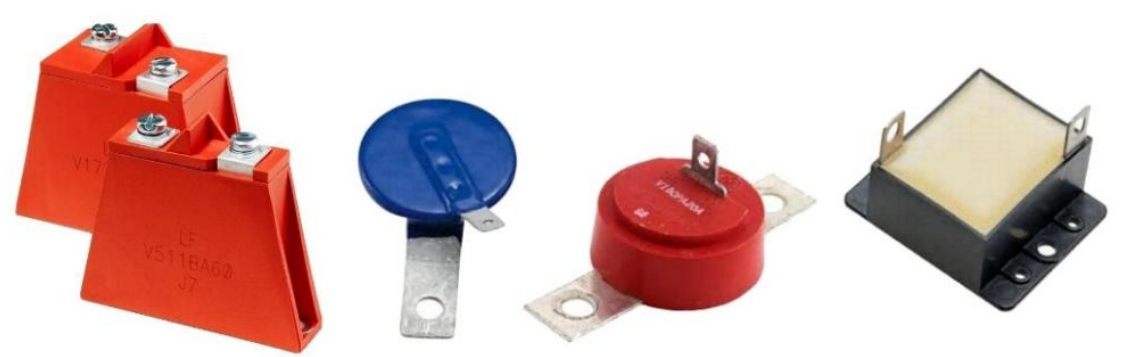

Fig7. Some types of powerful varistors that ensure protection of $A C$ and $D C$ circuits from high voltage transients.

The so called suppressors (TVS - transient voltage suppressors) based on avalanche diodes, Fig. 8, are more advantageous compared to varistors in terms of response time (which is very important in case of a short HEMP pulse).

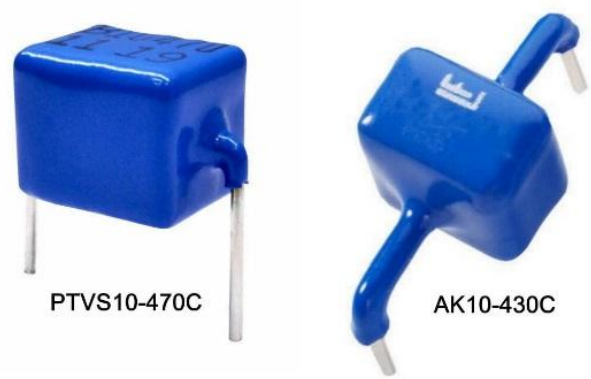

Fig8. Some types of fast powerful suppressors based on avalanche diodes.

Varistors and suppressors feature the so called clamping voltage, i.e. the voltage remaining on a varistor and the protected device connected to it in parallel after the varistor's actuation. As the common EMC standards require resilience to high voltage transient with the amplitude of $2.5-4 \mathrm{kV}$, the powerful varistors (or TVS-diodes) with the clamping voltage of 500-600 Volts will ensure reliable protection of equipment from HEMP impact without any high frequency HEMP filters. Why is that important?

Because:

- HEMP filters are expensive, especially powerful filters designed for high currents;

- in case of multiple circuits requiring protection, the standard approach will need many HEMP filters that occupy a lot of space, which is not often available.

- HEMP filters are connected into the cut of control and power cables, and in case of multiple circuits, this hampers filter installation and makes the work more expensive and more difficult.

Small and inexpensive varistors, protecting control and power circuits from HEMP impact connected parallel (rather than into the cable cut) to the protected devices, make the protection significantly simpler and cheaper.

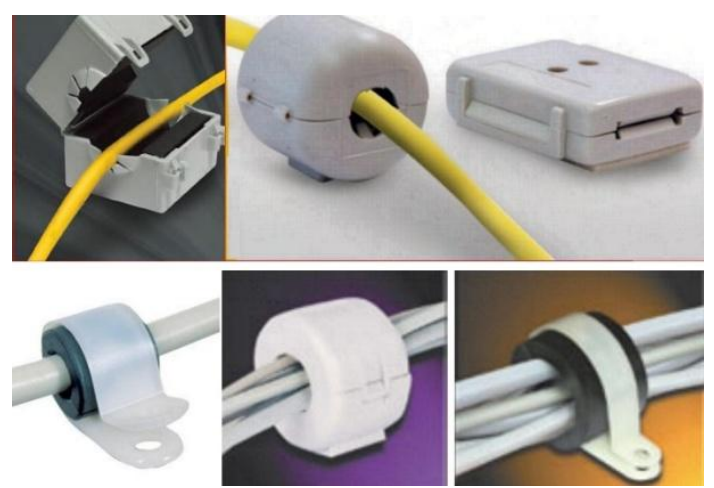

Fig9. Modular ferrite rings put onto the cable for additional protection of extra-sensitive electronic equipment from electromagnetic interference.

International Journal of Research Studies in Electrical and Electronics Engineering (IJRSEEE) Page | 34 
In addition, it must be stressed that most industrial electronic devices are already equipped with varistors in their input circuits, for example, power supply, logic inputs of relay protection, etc., Fig. 10. Sometimes, the power dissipation of such small varistors is not enough for protection against very high power HEMP, therefore many circuits must be protected with addition powerful varistors, for example current-carrying busbars $\mathrm{AC}$ and $\mathrm{DC}$, common power supplies, etc.

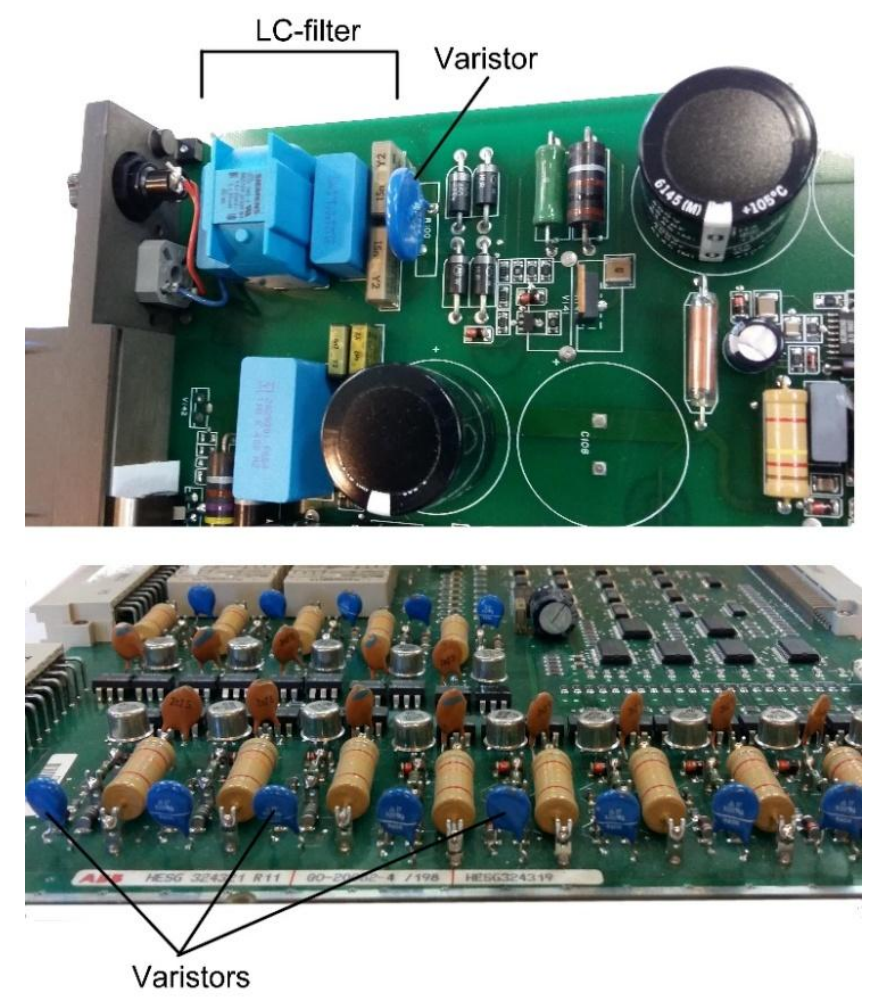

Fig10. Usage varistors in power supply (up) and logic inputs (down) of relay protection

Sometimes, when extra-sensitive equipment is used, it is possible to use modular ferrite rings (cylinders) that are put on the outside of the protected cable, Fig. 9 [12].

The problem can arise only when the protected object features very low internal resistance, such as accumulator battery. When a HEMP-generated voltage pulse is applied to this device, the voltage dip may be not sufficient for the varistor's actuation. However, the current flowing through such a device can be significant enough due to low internal resistance. In this case, an inductive choke with impedance negligible for a DC current, but high enough for a high-frequency signal (short pulse), should be connected in series with the protected device.

\section{Conclusions}

It is much more important for effective protection of equipment against HEMP to use correct transient voltage suppressing rather than high frequency filtering. The above mentioned approach means that regardless of the widespread approach to protection of electric and electronic equipment against HEMP, it is not necessary to employ special expensive filters acting as basic protection means. Alternatively, simple elements protecting from high voltage pulse (e.g. varistors or suppressors) will do a good job. Moreover, combination of the latter with other known protective means and methods will make protection of equipment from HEMP much easier and significantly cheaper.

\section{REFERENCES}

[1] Gurevich V. I. Intentional Electromagnetic Destructive Impacts is the Threat of National Security. - Issues of Risk Analysis, 2016, vol. 13, No. 5, pp. 44 -51.

[2] Gurevich V. Basic HEMP Protection Means for a Power Substation: Quick Guide. -International Journal of Electrical and Electronics Research (IJEER), 2017, Vol. 5, Issue 2, pp. 12 - 19.

[3] Gurevich V. EMP and Its Impact on Electrical Power System: Standards and Reports. International Journal of Research and Innovation in Applied Science (IJRIAS)", 2016, Vol I, Issue VI, pp. 5 - 10 . 
[4] MIL-STD-188-125-1 High-Altitude Electromagnetic Pulse (HEMP) Environmental Protection for Ground Based C41 Facilities Performing Critical. Time-Urgent Mission. Part 1Fixed Facilities, 2005.

[5] Report HDL-TM-81-9, AD-A100141. Applications of Filters for High-Altitude Electromagnetic Pulse Protection. - U. S. Army Electronics Research and Development Command, Harry Diamond Laboratories, 1981.

[6] High Voltage HEMP Filters. - API Technologies Corp. Proprietary Information, http://eis.apitech.com/docs/filmcaps/HV-HEMP-Filters.pdf.

[7] MPE Application Notes. HEMP Filter Maintenance and Monitoring, https://www.mpe.co.uk/wpcontent/uploads/2013/02/HEMP-Filter-Maintenance.pdf

[8] HEMP Filters, European EMC Products Ltd, http://www.euro-emc.co.uk/files/HEMPFilters.pdf.

[9] EMP/HEMP Protector/Filters. Requirements of MIL-STD-188-125-1 \& 2. - Holland Shielding Systems, https://faradayfilters.com/PDF_documents/PDF_EMP-HEMP\%20protectorfilters_November-12-2012-231pm.pdf.

[10] S. M. Han, C. S. Huh, J. S. Choi, A Validation of Conventional Protection Devices in Protecting EMP Threats. - Progress in Electromagnetics Research, vol. 119, 2011, pp. 253-263.

[11] S. M. Hwang, J. I. Hong, S. M. Han, The Susceptibility of Microcontroller Device with Coupling Caused by UWB-HPEM. - Progress in Electromagnetics Research Symposium Proceedings, Moscow, August 18-21, 2009, pp. 1269-1272.

[12] Gurevich V. I. Ferrite Filters. - Electrical Engineering \& Electromechanics, 2015, No. 5, pp. 69 74.

\section{AUTHOR'S BIOGRAPHY}

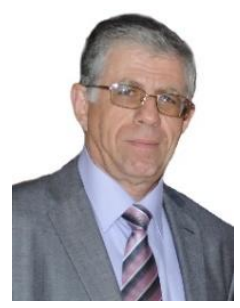

Vladimir I. Gurevich was born in Kharkov, Ukraine, in 1956. He received an M.S.E.E. degree (1978) at the Kharkov Technical University, named after P. Vasilenko, and a Ph.D. degree (1986) at Kharkov National Polytechnic University.

His employment experience includes: teacher, assistant professor and associate professor at Kharkov Technical University, and chief engineer and director of Inventor, Ltd.

In 1994, he arrived in Israel and works today at Israel Electric Corp. as a Senior Specialist and Head of section of the Central Electric Laboratory.

$\mathrm{He}$ is the author of more than 200 professional papers and 15 books and holder of nearly 120 patents in the field of electrical engineering and power electronics. In 2006 he was Honorable Professor with the Kharkov Technical University. 